\section{RMD Open}

Rheumatic \&

Musculoskeletal Diseases

\title{
Impact of tocilizumab monotherapy on patient-reported outcomes in patients with rheumatoid arthritis from two randomised controlled trials
}

\author{
Vibeke Strand, ${ }^{1}$ Margaret Michalska, ${ }^{2}$ Christine Birchwood, ${ }^{2}$ \\ Jinglan Pei, ${ }^{2}$ Katie Tuckwell, ${ }^{3}$ Rebecca Finch, ${ }^{9}$ Cem Gabay, ${ }^{10}$ Arthur Kavanaugh, ${ }^{11}$ \\ Graeme Jones ${ }^{12}$
}

To cite: Strand V, Michalska M, Birchwood C, et al. Impact of tocilizumab monotherapy on patient-reported outcomes in patients with rheumatoid arthritis from two randomised controlled trials. RMD Open 2017;3:e000496. doi:10.1136/ rmdopen-2017-000496

- Prepublication history and additional material for this paper are available online. To view please visit the journal (http://dx doi.org/10.1136/rmdopen-2017000496).

Received 12 May 2017 Revised 7 August 2017 Accepted 11 August 2017

CrossMark

For numbered affiliations see end of article.

\section{ABSTRACT}

Objective Two randomised controlled trials, AMBITION (NCT00109408) and ADACTA (NCT01119859), showed tocilizumab (TCZ) monotherapy superior to methotrexate (MTX) and adalimumab (ADA) monotherapy, respectively, for improving rheumatoid arthritis (RA) disease activity. This study compared the benefit of TCZ versus MTX or ADA monotherapy for improving patient-reported outcomes (PROs) in patients with RA.

Methods PROs included patient global assessment (PtGA), pain, Health Assessment Questionnaire Disability Index (HAQ-DI), Functional Assessment of Chronic IIIness Therapy (FACIT)-Fatigue and Short Form-36 (SF-36) physical component summary (PCS) and mental component summary (MCS) and eight domain scores. Outcomes included proportions of patients reporting changes from baseline in PRO scores $\geq$ minimum clinically important differences (MCID) and zage-matched and gendermatched normative values at 24 weeks.

Results In AMBITION, TCZ-treated patients reported significantly greater mean improvements in $\mathrm{HAQ}(-0.7$ vs -0.5$)$, FACIT-Fatigue (8.7 vs 5.7), SF-36 PCS ( 9.8 vs 7.8) and five SF-36 domains at week 24 than with MTX; $45.0 \%-84.0 \%$ of TCZ-treated patients reported improvements $\geq \mathrm{MCID}$, and $24.3 \%-52.1 \%$ reported scores $\geq$ normative values across all PROs versus $39.4 \%$ $81.8 \%$ and $14.5 \%-45.0 \%$, respectively, with MTX. In ADACTA, TCZ-treated patients reported significantly greater improvements in PtGA (-42.3 vs -31.8$)$, pain (-40.1 vs $-28.7)$, SF-36 MCS (7.9 vs 5.0) and three SF-36 domains than with ADA; $57.7 \%-83.3 \%$ of TCZ-treated patients reported improvements $\geq \mathrm{MCID}$, and $22.1 \%-49.3 \%$ reported scores $\geq$ normative values across all PROs versus $13.6 \%-37.8 \%$, respectively, with ADA.

Conclusions TCZ monotherapy resulted in more patients reporting clinically meaningful PRO improvements and PRO scores $\geq$ normative values compared with MTX or ADA monotherapy.

Trial registration numbers NCT00109408 and NCT01119859; Post-results.

\section{Key messages}

What is already known about this subject?

- Tocilizumab (TCZ) monotherapy was shown superior to methotrexate (MTX) monotherapy and adalimumab (ADA) monotherapy in two randomised, controlled trials (AMBITION and ADACTA, respectively) for improving disease activity in patients with active rheumatoid arthritis $(\mathrm{RA})$; however, there are limited data regarding the impact of TCZ monotherapy on patient-reported outcomes (PROs).

What does this study add?

- In this post hoc analysis of the AMBITION and ADACTA trial populations, treatment with TCZ, MTX or ADA as monotherapy resulted in substantial and clinically meaningful improvements in PROs, including patient global assessment, pain, Health Assessment Questionnaire Disability Index, Functional Assessment of Chronic IIIness TherapyFatigue and Short Form-36 physical and mental component summary and eight domain scores, over 24 weeks.

- TCZ monotherapy resulted in greater mean improvements from baseline in PRO scores and more patients reporting clinically meaningful PRO improvements and PRO scores $\geq$ age-matched and gender-matched normative values compared with MTX or ADA monotherapy.

How might this impact on clinical practice?

- Treatment with TCZ, MTX or ADA monotherapy was effective in improving PROs, including healthrelated quality of life, in patients with active RA; however, TCZ monotherapy was more effective overall compared with MTX or ADA monotherapy.

- Results of these trials indicate that it is now possible for patients with RA to achieve PRO scores that more closely approach those reported by healthy populations. 


\section{INTRODUCTION}

Rheumatoid arthritis (RA) is a systemic autoimmune disorder characterised by inflammation of the joints. Patients with RA often experience diminished health-related quality of life (HRQOL) with respect to both physical functioning and emotional state due to the pain, stiffness, fatigue and disability that can result from this inflammation. ${ }^{1-4}$ The goal of treatment in patients with RA is to reduce disease activity and improve patients' HRQOL. Patient-reported outcomes (PROs) are important measures when determining response to therapy in patients with RA, and patients report that, from their perspective, these measures of HRQOL are more important than traditional measures of clinical disease activity. ${ }^{5-9}$

Methotrexate (MTX) is the recommended first-line treatment for patients with RA. ${ }^{10}$ For patients with inadequate responses to MTX, addition of biological therapy in conjunction with MTX is recommended. ${ }^{10}$ However, approximately one-third of patients with RA who receive biologics do so as monotherapy, most often due to intolerance of or contraindications to MTX or by patient choice to reduce personal medication burden without physician consultation. ${ }^{11}{ }^{12}$ It is therefore necessary to evaluate the efficacy of biological monotherapy for improvement of both clinical disease activity and PROs in patients with RA.

Tocilizumab (TCZ) is a monoclonal antibody that inhibits the interleukin-6 receptor and is approved for the treatment of patients with moderate to severe RA. Previous randomised controlled trials (RCTs) have demonstrated the efficacy of TCZ, both as monotherapy and in combination with conventional synthetic disease-modifying antirheumatic drugs, such as MTX, for improvement of disease activity in patients with RA. ${ }^{13}{ }^{14}$ In addition, in two RCTs, AMBITION and ADACTA, respectively, TCZ monotherapy was shown superior to MTX monotherapy and monotherapy with the tumour necrosis factor inhibitor (TNFi) adalimumab (ADA)..$^{15} 16$

In a phase 3 RCT, TCZ with concomitant MTX was shown to significantly improve PROs over 24 weeks compared with placebo in patients with RA who were inadequate responders to TNFis. ${ }^{17}$ However, there are limited data regarding the impact of TCZ monotherapy on PROs. The objective of this study was to compare the efficacy of TCZ monotherapy with that of MTX or ADA monotherapy for improvement in PROs in patients with RA based on post hoc analyses of AMBITION (NCT00109408) and ADACTA (NCT01119859). ${ }^{15} 16$

\section{METHODS}

\section{Study design and patient population}

The study designs and patient inclusion and exclusion criteria for both RCTs have been previously described and are summarised in online supplementary table S1. Briefly, AMBITION was a phase 3 multicentre RCT that compared the efficacy of TCZ monotherapy with that of MTX monotherapy in patients with moderate to severely active RA. ${ }^{16}$ Eligible patients were MTX naive or had discontinued MTX $\geq 6$ months prior to randomisation and were not inadequate responders to MTX (MTXIR) or TNFis. Study participants received TCZ $8 \mathrm{mg} /$ $\mathrm{kg}$ intravenous every 4 weeks as monotherapy or MTX $7.5-20 \mathrm{mg}$ /week as monotherapy.

ADACTA was a phase 4 multicentre RCT that compared the efficacy of TCZ monotherapy with that of ADA monotherapy in patients with RA. ${ }^{15}$ Patients had severe active RA, were biologic naïve and MTX-IR or otherwise inappropriate candidates for continued MTX treatment by judgement of the investigator. Study participants received TCZ $8 \mathrm{mg} / \mathrm{kg}$ intravenous every 4 weeks or ADA $40 \mathrm{mg}$ subcutaneous (SC) every 2 weeks. At week 16 , or any time thereafter, patients in both treatment arms with $<20 \%$ improvement in swollen and tender joint counts were eligible for escape treatment with weekly SC injections (ADA and placebo).

\section{Patient-reported outcomes}

HRQOL was assessed at baseline and 24 weeks in each study population. PROs assessed included patient global assessment (PtGA; visual analogue scale (VAS), 0-100 mm); pain (VAS, 0-100 mm); Health Assessment Questionnaire Disability Index (HAQ-DI; 0-3); Functional Assessment of Chronic Illness Therapy (FACIT)-Fatigue (0-52); Short Form-36 (SF-36) physical component summary (PCS) and mental component summary (MCS) scores (mean: 50, SD: 10); and eight domains (physical functioning, role-physical, bodily pain, general health, vitality, social functioning, role-emotional and mental health; scored 0-100). Study outcomes included mean changes from baseline in PROs, the proportion of patients who reported improvements from baseline $\geq$ minimum clinically important differences (MCID) for each $\mathrm{PRO}^{18}{ }^{19}$ and the proportion of patients who reported scores zage-matched and gender-matched normative values (table 1). ${ }^{18-20}$ Mean SF-36 domain scores were determined at baseline and 24 weeks and compared with age-matched and gender-matched normative values for each study population using spydergrams. ${ }^{21}$ Changes from baseline in Clinical Disease Activity Index (CDAI) were assessed at 24 weeks as a reference for change in disease activity.

\section{Statistical analysis}

Analyses were performed in the primary efficacy patient populations in each trial. In AMBITION, the primary efficacy hypothesis was to establish non-inferiority of TCZ versus MTX in the per-protocol population (TCZ, $n=265$ of 286 intention-to-treat (ITT) patients; MTX, $\mathrm{n}=259$ of 284 ITT patients). In ADACTA, the primary efficacy hypothesis was to establish superiority of TCZ versus ADA in the ITT population (TCZ, $\mathrm{n}=163$; ADA, $\mathrm{n}=162$ ). PROs, the proportions of patients reporting improvements $\geq$ MCID from baseline to week 24 and those reporting scores $\geq$ age-matched and gender-matched normative values at week 24 were compared between TCZ and MTX 
Table 1 PRO age-matched and gender-matched normative values in non-RA population without comorbid conditions

\begin{tabular}{lll}
\hline & AMBITION & ADACTA \\
\hline HAQ-DI, 0-3 & $<0.5$ & $<0.5$ \\
FACIT-Fatigue, 0-52 & $\geq 40$ & $\geq 40$ \\
SF-36 PCS (mean:50, SD: 10) & $\geq 50$ & $\geq 50$ \\
SF-36 MCS (mean: 50, SD:10) & $\geq 50$ & $\geq 50$ \\
SF-36 domains, 0-100 & & \\
Physical functioning & $\geq 78.8$ & $\geq 78.3$ \\
Role-physical & $\geq 79.1$ & $\geq 79.0$ \\
Bodily pain & $\geq 67.4$ & $\geq 68.1$ \\
General health & $\geq 68.2$ & $\geq 69.3$ \\
Vitality & $\geq 56.6$ & $\geq 58.3$ \\
Social functioning & $\geq 81.7$ & $\geq 83.4$ \\
Role-emotional & $\geq 85.0$ & $\geq 86.3$ \\
Mental health & $\geq 72.9$ & $\geq 75.1$ \\
\hline
\end{tabular}

FACIT, Functional Assessment of Chronic Illness Therapy; HAQ-DI, Health Assessment Questionnaire Disability Index; MCS, mental component summary; PCS, physical component summary; PRO, patient-reported outcome; RA, rheumatoid arthritis; SF-36, Short Form-36.

or ADA in AMBITION and ADACTA, respectively. For patients in ADACTA who received escape therapy and completed the study to 24 weeks (TCZ, $n=7 ; \mathrm{ADA}, \mathrm{n}=8$ ), results were carried forward from the time of escape. In AMBITION, p values were not reported, as non-inferiority was determined from the lower limit of the $95 \%$ CI for the treatment difference (TCZ minus MTX); if the lower limit of the $95 \%$ CI for the treatment difference was $>0$, then superiority was achieved. In ADACTA, $p$ values were reported to determine statistically significant differences between TCZ and ADA.

Continuous endpoints were compared using least squares mean changes from baseline calculated using an analysis of covariance. The proportion of patients reporting improvements from baseline $\geq$ MCID at 24 weeks was analysed for each PRO and SF-36 domain using a Cochran-Mantel-Haenszel $\chi^{2}$ test. All analyses were adjusted for site (AMBITION)/region (ADACTA), baseline scores (ADACTA) and duration of RA.

\section{RESULTS}

\section{Baseline patient characteristics}

Patient demographics and baseline disease characteristics have been previously described, were generally comparable between treatment groups within each RCT and showed that patients were substantially impacted by their disease (table 2) ${ }^{15}{ }^{16}$ In the MTX and TCZ arms in AMBITION, $81 \%$ and $83 \%$ of patients were women, $73 \%$ and $71 \%$ white, mean age was 50.1 and 51.1 years and mean disease duration 6.3 and 6.4 years, respectively; mean baseline CDAI was 43.2 in both arms. In the ADA and TCZ arms in ADACTA, $82 \%$ and $79 \%$ of patients were women, $82 \%$ and $89 \%$ white, mean age was 53.3 and 54.4 years, mean disease duration 6.3 and 7.3 years and mean baseline CDAI 43.1 and 40.8, respectively.

\section{Improvement in PROs at 24 weeks}

Patients who received TCZ monotherapy in both RCTs reported greater improvements from baseline across all PROs at 24 weeks than those who received MTX or ADA monotherapy. In AMBITION, TCZ-treated patients reported significantly greater improvements from baseline in HAQ-DI, FACIT-Fatigue and SF-36 PCS scores at 24 weeks than MTX-treated patients (table 3). In addition, TCZ-treated patients reported significantly greater improvements from baseline in five of eight SF-36 domains (physical functioning, bodily pain, vitality, social functioning and mental health) than MTX-treated patients. In ADACTA, patients who received TCZ reported significantly greater improvements from baseline in PtGA, pain and SF-36 MCS scores at 24 weeks than those who received ADA (table 3). TCZ-treated patients also reported significantly greater improvements from baseline in three of eight SF-36 domains (role-physical, vitality and social functioning) than ADA-treated patients.

Patients who received TCZ monotherapy in both RCTs reported higher mean scores across all SF-36 domains, which more closely approached age-matched and gendermatched normative values, at 24 weeks than patients who received MTX or ADA monotherapy (figure 1), indicative of clinically meaningful improvements. Consistent with reported improvements in PROs, patients treated with TCZ monotherapy in either RCT experienced significantly greater improvements from baseline in CDAI at 24 weeks than patients who received MTX or ADA monotherapy (table 3 ).

\section{Patients reporting improvements $\geq M C I D$ at 24 weeks}

At least one patient in all treatment groups reported improvements $\geq$ MCID across all PROs. In AMBITION, significantly more patients who received TCZ monotherapy reported improvements from baseline $\geq$ MCID in HAQ-DI (number needed to treat (NNT): 11.0), FACIT-Fatigue (NNT: 7.8), SF-36 role-physical (NNT: 10.9) and vitality (NNT: 14.5) domains at 24 weeks than patients who received MTX monotherapy (figure 2A; online supplementary table S2). In ADACTA, a significantly higher proportion of patients who received TCZ monotherapy reported clinically meaningful improvements from baseline in pain (NNT: 7.5), SF-36 MCS (NNT: 6.4) and SF-36 vitality domain (NNT: 6.0) scores at 24 weeks compared with patients who received ADA monotherapy (figure 2B; online supplementary table S2).

Patients reporting scores $\geq$ age-matched and gender-matched normative values at 24 weeks

The proportions of patients reporting scores zage-matched and gender-matched normative values at baseline were 
Table 2 Baseline demographics, disease characteristics and PRO scores of patients in AMBITION and ADACTA

\begin{tabular}{|c|c|c|c|c|}
\hline & AMBITION & & ADACTA & \\
\hline Baseline characteristic ${ }^{*}$ & TCZ $8 \mathrm{mg} / \mathrm{kg}(\mathrm{n}=265)$ & MTX 7.5-20 mg (n=259) & TCZ $8 \mathrm{mg} / \mathrm{kg}(\mathrm{n}=163)$ & ADA $40 \mathrm{mg}(\mathrm{n}=162)$ \\
\hline Age, years & $51.1(13.1)$ & $50.1(12.8)$ & $54.4(13.0)$ & $53.3(12.4)$ \\
\hline Female, n (\%) & $219(83)$ & $211(81)$ & $129(79)$ & $133(82)$ \\
\hline White, n (\%) & $187(71)$ & $188(73)$ & $145(89)$ & $133(82)$ \\
\hline Disease duration, years & $6.4(7.7)$ & $6.3(7.9)$ & $7.3(8.1)$ & $6.3(6.9)$ \\
\hline Number of prior DMARDs & $1.2(1.3)$ & $1.1(1.4)$ & $2.0(1.1)$ & $2.0(1.1)$ \\
\hline CDAI & $43.2(12.9)$ & $43.2(11.8)$ & $40.8(12.3)$ & $43.1(12.6)$ \\
\hline PtGA, VAS 0-100 mm & $64.0(21.5)$ & $65.4(19.5)$ & $71.2(20.8)$ & $73.4(19.4)$ \\
\hline Pain, VAS 0-100 mm & $59.2(22.5)$ & $61.3(20.4)$ & $67.2(21.3)$ & $67.9(20.7)$ \\
\hline HAQ-DI, 0-3 & $1.6(0.7)$ & $1.5(0.6)$ & $1.6(0.6)$ & $1.7(0.6)$ \\
\hline FACIT-Fatigue, 0-52 & $27.4(10.6)$ & $27.8(10.5)$ & $24.9(10.6)$ & $24.1(11.2)$ \\
\hline SF-36 PCS (mean: 50, SD: 10) & $31.9(7.5)$ & $31.1(6.9)$ & $30.5(7.9)$ & $30.2(7.9)$ \\
\hline SF-36 MCS (mean: 50, SD: 10) & $40.2(12.0)$ & $40.6(11.3)$ & $39.7(12.0)$ & $38.9(12.3)$ \\
\hline \multicolumn{5}{|l|}{ SF-36 domains, $0-100$} \\
\hline Physical functioning & $37.1(24.1)$ & $37.0(23.2)$ & $34.4(22.0)$ & $32.9(24.0)$ \\
\hline Role-physical & $13.6(26.8)$ & $13.3(28.2)$ & $34.0(20.9)$ & $35.4(24.2)$ \\
\hline Bodily pain & $29.1(17.2)$ & $27.6(15.2)$ & $27.2(19.1)$ & $24.5(16.7)$ \\
\hline General health & $42.1(19.9)$ & $40.1(19.6)$ & $42.5(19.4)$ & $40.6(18.6)$ \\
\hline Vitality & $35.7(19.7)$ & $37.0(19.1)$ & $32.7(18.1)$ & $32.8(19.5)$ \\
\hline Social functioning & $48.3(26.4)$ & $50.2(24.8)$ & $48.0(26.9)$ & $47.7(26.8)$ \\
\hline Role-emotional & $34.9(41.7)$ & $32.7(42.8)$ & $54.4(30.7)$ & $50.3(31.7)$ \\
\hline Mental health & $55.5(22.0)$ & $57.7(20.3)$ & $54.9(19.5)$ & 54.1 (20.9) \\
\hline
\end{tabular}

${ }^{*}$ All values are presented as mean (SD) unless otherwise indicated.

ADA, adalimumab; CDAI, Clinical Disease Activity Index; DMARDs, disease-modifying antirheumatic drugs; FACIT, Functional Assessment of Chronic Illness Therapy; HAQ-DI, Health Assessment Questionnaire Disability Index; MCS, mental component summary; MTX, methotrexate; PCS, physical component summary; PRO, patient-reported outcome; PtGA, patient global assessment; SF-36, Short Form-36;

TCZ, tocilizumab; VAS, visual analogue scale.

comparable between treatment groups in both RCTs. In AMBITION, the proportion of patients with normative scores at baseline ranged from $0.8 \%$ and $1.5 \%$ (SF-36 PCS; MTX and TCZ, respectively) to $23.6 \%$ and $24.2 \%$ (SF-36 MCS; TCZ and MTX, respectively), with a similar range across SF-36 domains: $1.2 \%$ and $3.4 \%$ (bodily pain; MTX and TCZ, respectively) to $24.4 \%$ and $25.3 \%$ (role-emotional; TCZ and MTX, respectively). In ADACTA, the proportion of patients with normative scores at baseline ranged from $1.9 \%$ and $2.5 \%$ (SF-36 PCS; TCZ and ADA, respectively) to $20.8 \%$ and $21.1 \%$ (SF-36 MCS; ADA and TCZ, respectively), with a similar range across SF-36 domains: $1.2 \%$ (role-physical; TCZ) and $2.5 \%$ (bodily pain; ADA) to $17.4 \%$ and $19.1 \%$ (role-emotional; TCZ and ADA, respectively).

The proportion of patients reporting scores $\geq$ age-matched and gender-matched normative values at 24 weeks was greater than at baseline for all treatment groups across all PROs and indicated clinically important improvements in TCZ-treated patients (figure 3). In AMBITION, $24 \%-44 \%$ of TCZ-treated patients reported scores $\geq$ normative values across HAQ-DI, FACIT-Fatigue and SF-36 PCS/MCS and 30\%-52\% across SF-36 domains at week 24 compared with $15 \%-42 \%$ and $21 \%-41 \%$ of MTX-treated patients, respectively. In ADACTA, the proportion of TCZ-treated patients reporting scores $\geq$ normative values ranged from $22 \%$ to $49 \%$ for HAQ-DI, FACIT-Fatigue and SF-36 PCS/MCS and 23\% to $41 \%$ across SF-36 domains at week 24 compared with $14 \%-38 \%$ and $18 \%-33 \%$ of ADA-treated patients, respectively.

\section{DISCUSSION}

Consistent with CDAI responses in AMBITION and ADACTA, TCZ monotherapy was more effective improving PROs in patients with active RA than either MTX or ADA monotherapy. Although patients treated with MTX or ADA reported clinically meaningful improvement in PROs, patients who received TCZ reported significantly greater improvements from baseline at 24 weeks than patients who received either MTX or ADA. Similarly, a higher proportion of TCZ-treated patients reported improvements from baseline $\geq \mathrm{MCID}$ as well as scores $\geq$ age-matched and gender-matched normative values, indicative of clinically meaningful changes, at 24 weeks than patients treated with either MTX or ADA. 
Table 3 LSM changes from baseline in PROs at 24 weeks in AMBITION and ADACTA

\begin{tabular}{|c|c|c|c|c|c|c|c|}
\hline & \multicolumn{3}{|c|}{ AMBITION* } & \multicolumn{4}{|c|}{ ADACTA* } \\
\hline & \multicolumn{2}{|c|}{$\begin{array}{l}\text { LSM change from } \\
\text { baselinet }\end{array}$} & \multirow[b]{2}{*}{ Difference $(95 \% \mathrm{Cl})$} & \multicolumn{2}{|c|}{$\begin{array}{l}\text { LSM change from } \\
\text { baseline† }\end{array}$} & \multirow[b]{2}{*}{ Difference $(95 \% \mathrm{Cl})$} & \multirow[b]{2}{*}{ p value } \\
\hline & TCZ & MTX & & TCZ & ADA & & \\
\hline $\begin{array}{l}\text { PtGA, } \\
\text { VAS 0-100 mm }\end{array}$ & -33.5 & -29.5 & $-4.1(-9.3$ to 1.2$)$ & -42.3 & -31.8 & $-10.5(-17.7$ to -3.3$)$ & 0.004 \\
\hline $\begin{array}{l}\text { Patient pain, } \\
\text { VAS 0-100 } \mathrm{mm}\end{array}$ & -31.5 & -29.5 & $-2.0(-7.1$ to 3.1$)$ & -40.1 & -28.7 & $-11.3(-18.3$ to -4.3$)$ & 0.002 \\
\hline HAQ-DI, 0-3 & $-0.7 \ddagger$ & -0.5 & $-0.2(-0.3$ to -0.1$)$ & -0.7 & -0.5 & $-0.2(-0.3$ to 0.0$)$ & 0.065 \\
\hline FACIT-Fatigue, 0-52 & $8.7 \ddagger$ & 5.7 & 2.9 (1.0 to 4.8$)$ & 11.4 & 8.9 & $2.5(-0.3$ to 5.3$)$ & 0.077 \\
\hline $\begin{array}{l}\text { SF-36 PCS (mean: 50, SD: } \\
10)\end{array}$ & $9.8 \ddagger$ & 7.8 & $2.0(0.4$ to 3.7$)$ & 9.2 & 7.6 & $1.6(-0.6$ to 3.8$)$ & 0.164 \\
\hline $\begin{array}{l}\text { SF-36 MCS (mean: 50, SD: } \\
10)\end{array}$ & 6.8 & 4.8 & $2.0(-0.3$ to 4.2$)$ & 7.9 & 5.0 & $2.9(0.0$ to 5.9$)$ & 0.050 \\
\hline \multicolumn{8}{|l|}{$\begin{array}{l}\text { SF-36 domains, } \\
0-100\end{array}$} \\
\hline Physical functioning & 23.0‡ & 16.5 & 6.5 (2.2 to 10.8$)$ & 20.0 & 16.0 & $4.0(-1.5$ to 9.5$)$ & 0.156 \\
\hline Role-physical & 37.3 & 29.8 & $7.5(-0.1$ to 15.2$)$ & 23.8 & 16.4 & 7.4 (1.3 to 13.5$)$ & 0.017 \\
\hline Bodily pain & $30.4 \ddagger$ & 24.8 & 5.7 (1.9 to 9.5$)$ & 29.3 & 23.8 & $5.5(-0.2$ to 11.2$)$ & 0.058 \\
\hline General health & 12.7 & 9.6 & $3.1(-0.1$ to 6.3$)$ & 10.7 & 7.6 & $3.0(-1.2$ to 7.3$)$ & 0.156 \\
\hline Vitality & $19.5 \ddagger$ & 12.9 & 6.6 (2.9 to 10.4$)$ & 19.1 & 14.0 & 5.1 (0.2 to 9.9$)$ & 0.040 \\
\hline Social functioning & $21.5 \ddagger$ & 16.4 & 5.1 (1.0 to 9.3$)$ & 23.4 & 17.1 & 6.3 (0.6 to 12.0$)$ & 0.032 \\
\hline Role-emotional & 28.5 & 22.2 & $6.3(-1.5$ to 14.1$)$ & 15.4 & 9.7 & $5.7(-0.6$ to 12.0$)$ & 0.077 \\
\hline Mental health & 14.3‡ & 10.2 & 4.1 (0.8 to 7.5$)$ & 13.5 & 9.3 & $4.2(-0.1$ to 8.5$)$ & 0.057 \\
\hline CDAI & $-25.6 \S$ & -19.8 & $-5.8(-8.6$ to -3.0$)$ & -23.8 & -18.9 & $-4.9(-8.3$ to -1.5$)$ & 0.005 \\
\hline
\end{tabular}

${ }^{*}$ Analyses were performed using the per-protocol population in AMBITION (TCZ, n=265; MTX, $n=259$ ) and the intention-to-treat population in ADACTA (TCZ, $n=163 ;$ ADA, $n=162)$.

†Adjusted for site (AMBITION)/region (ADACTA), baseline score (ADACTA) and duration of RA.

\#Statistical significance is demonstrated by the lower limit of the $95 \% \mathrm{Cl}$ of TCZ - MTX $>0$.

§Statistical significance is demonstrated by the upper limit of the $95 \% \mathrm{Cl}$ of TCZ $-\mathrm{MTX}<0$.

ADA, adalimumab; CDAI, Clinical Disease Activity Index; FACIT, Functional Assessment of Chronic Illness Therapy; HAQ-DI, Health Assessment Questionnaire Disability Index; LSM, least squares mean; MCS, mental component summary; MTX, methotrexate; PCS, physical component summary; PRO, patient-reported outcome; PtGA, patient global assessment; RA, rheumatoid arthritis; SF-36, Short Form-36;

TCZ, tocilizumab; VAS, visual analogue scale.

Patients in both RCT populations were substantially impacted by their disease at baseline, indicated by mean PRO scores below normative values in both trials and $<25 \%$ of patients in AMBITION and $<20 \%$ of patients in ADACTA reporting scores $\geq$ normative values in any PRO. The greater proportions of patients reporting normative scores at baseline in AMBITION versus ADACTA likely reflect a greater impact of disease in the biologic-eligible (ADACTA) versus an MTX-naïve (AMBITION) population. Treatment with TCZ, MTX or ADA monotherapy resulted in clinically meaningful improvements across all PROs. Although NNTs are typically generated in comparison with placebo treatment, NNTs based on HAQ-DI, FACIT and SF-36 physical functioning domain score differences in AMBITION and pain and SF-36 vitality domain score differences in ADACTA, despite active comparisons rather than placebo, were clinically meaningful $(\leq 10)$ favouring TCZ monotherapy. Additionally, higher proportions of patients in all treatment groups reported scores $\geq$ normative values at 24 weeks compared with baseline, indicative of clinically important improvements. These data indicate that achievement of normative PRO scores that more closely match those reported by healthy populations is an attainable goal for treatment of RA, regardless of therapy.

TCZ monotherapy resulted in improvements $\geq$ MCID in $\geq 1$ patient across all PROs in both studies, and a similar proportion of patients reported scores $\geq$ normative values at week 24 (AMBITION, 21\%-52\%; ADACTA, $22 \%-49 \%$ ) despite differences in prior treatment experiences between patients enrolled in AMBITION versus ADACTA. Thus, TCZ monotherapy was effective improving HRQOL in patients with active RA who had not experienced failure of MTX or TNFi therapy (AMBITION) and was effective as a first-line biologic in patients deemed inappropriate candidates for continued treatment with MTX (ADACTA). 

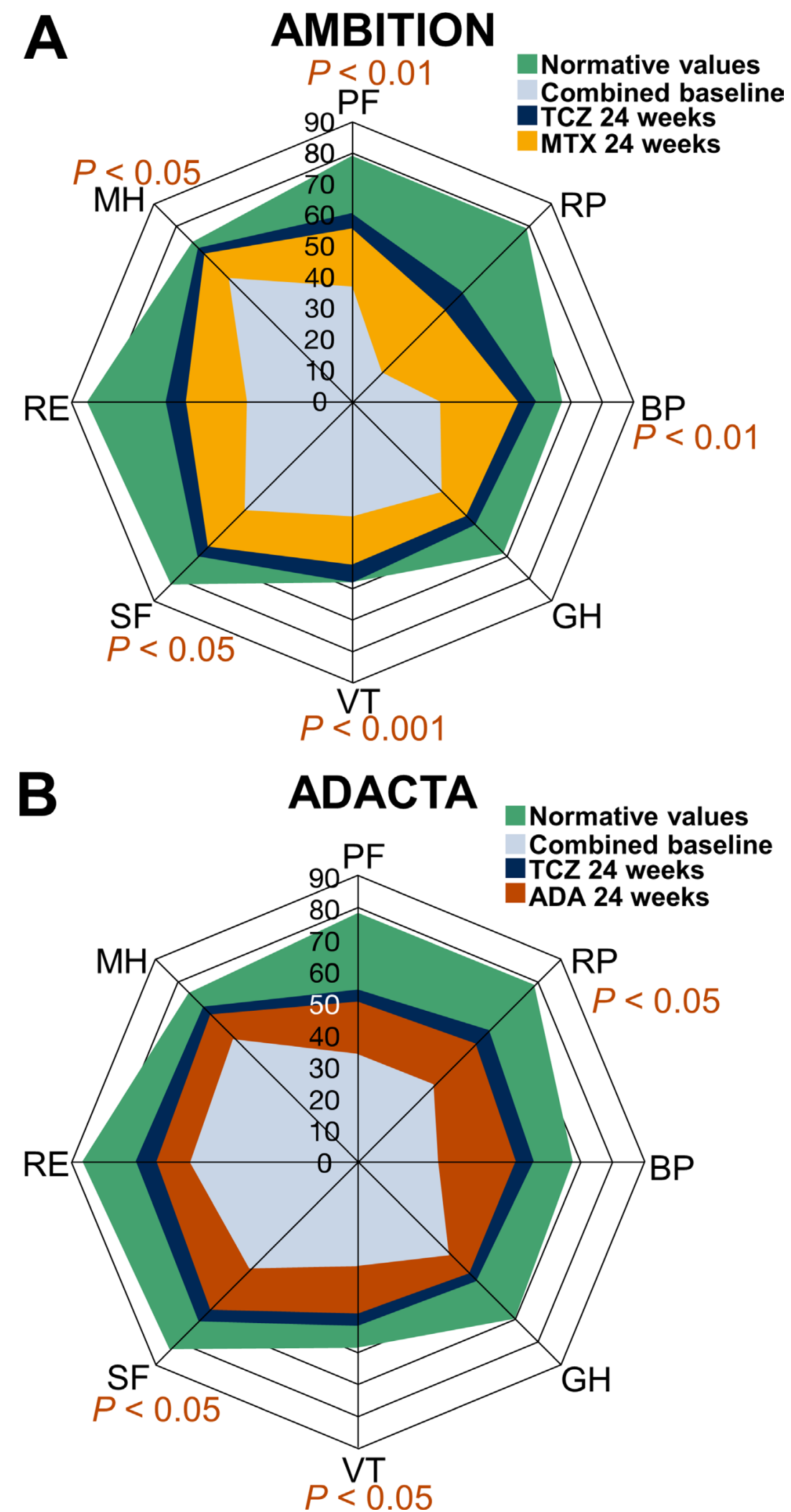

Figure 1 SF-36 domain scores at baseline and 24 weeks compared with age-matched and gender-matched normative values in the (A) AMBITION and (B) ADACTA trial populations. Analyses were performed using the per-protocol population in AMBITION (TCZ, $n=265$; MTX, $n=259$ ) and the intention-to-treat population in ADACTA (TCZ, $n=163 ; A D A, n=162$ ). Normative values were defined as age-matched and gender-matched scores in a non-RA population without comorbid conditions. AMBITION population: PF: $\geq 78.8$; RP: $\geq 79.1$; BP: $\geq 67.4$; GH: $\geq 68.2$; VT: $\geq 56.6$; SF: $\geq 81.7$; RE: $\geq 85.0 ; \mathrm{MH}: \geq 72.9$. ADACTA population: PF: $\geq 78.3$; RP: $\geq 79.0$; BP: $\geq 68.1$; GH: $\geq 69.3$; VT: $\geq 58.3$; SF: $\geq 83.4$; RE: $\geq 86.3$; $\mathrm{MH}: \geq 75.1$. ADA, adalimumab; BP, bodily pain; GH, general health; MH, mental health; MTX, methotrexate; PF, physical functioning; RA, rheumatoid arthritis; RE, role-emotional; RP, role-physical; SF, social functioning; SF-36, Short Form-36; TCZ, tocilizumab; VT, vitality. 
PROs

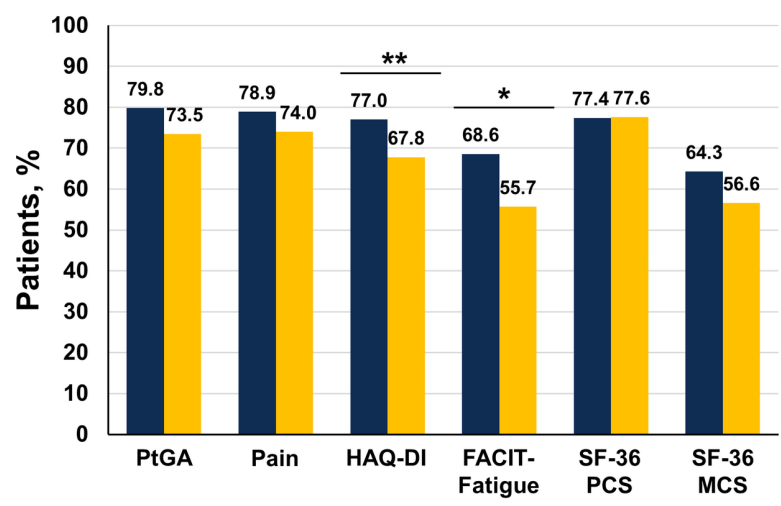

SF-36 Domains

TCZ

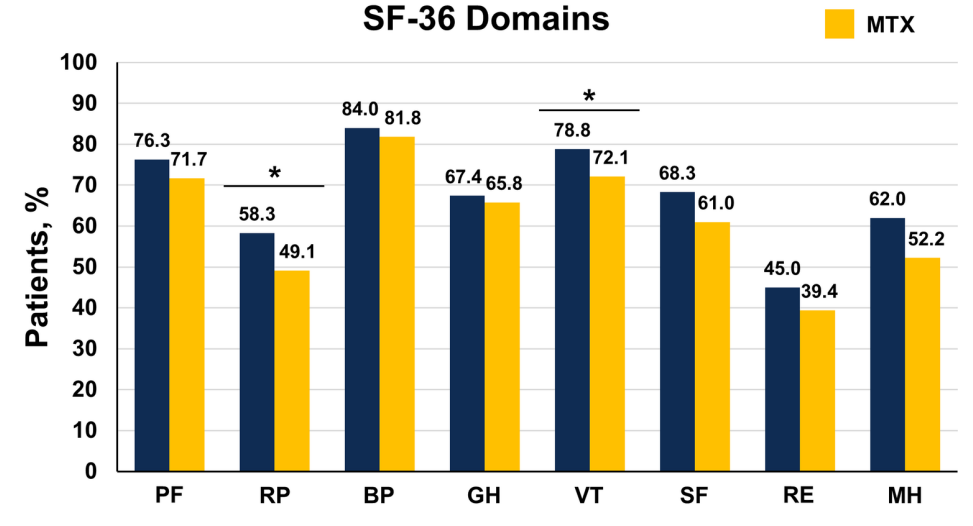

B

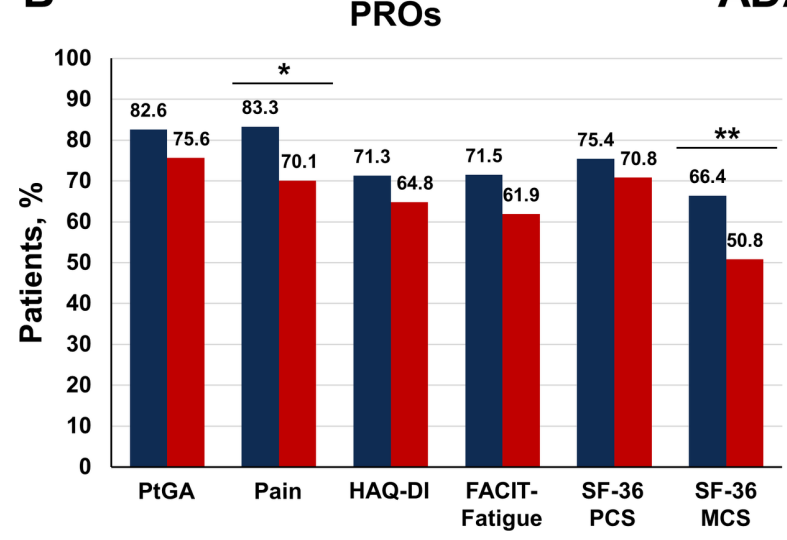

ADACTA
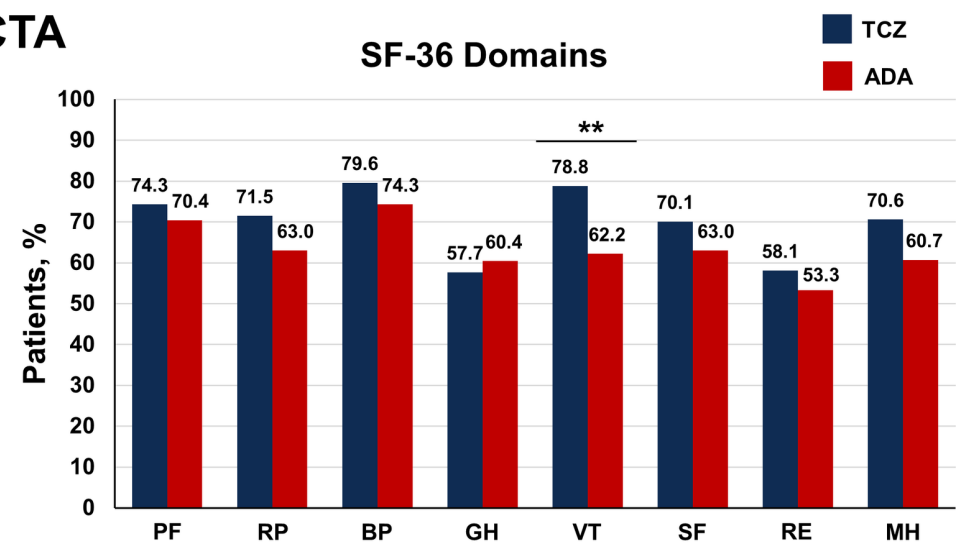

Figure 2 Proportion of patients reporting improvement $\geq M C I D$ at 24 weeks in the (A) AMBITION and (B) ADACTA trial populations. Analyses were performed using the per-protocol population in the AMBITION (TCZ, n=265; MTX, $n=259$ ) and the intention-to-treat population in ADACTA (TCZ, n=163; ADA, $n=162$ ) and adjusted for site (AMBITION)/region (ADACTA), baseline score (ADACTA) and duration of RA. The MCID for PROs were defined as follows: HAQ-DI: $\geq 0.22$; PtGA: $\geq 10$; patient pain: $\geq 10$; FACIT-Fatigue: $\geq 4$; SF-36 PCS/MCS: $\geq 2.5$; SF-36 domains: $\geq 5.0$. ADA, adalimumab; BP, bodily pain; FACIT, Functional Assessment of Chronic IIIness Therapy; GH, general health; HAQ-DI, Health Assessment Questionnaire Disability Index; MCS, mental component summary; MCID, minimum clinically important differences; MH, mental health; MTX, methotrexate; PCS, physical component summary; PF, physical functioning; PROs, patient-reported outcomes; PtGA, patient global assessment; $\mathrm{RE}$, role-emotional; RP, role-physical; SF, social functioning; SF-36, Short Form-36; TCZ, tocilizumab; VT, vitality. ${ }^{*} \mathrm{p}<0.05$; ${ }^{* *} \mathrm{p}<0.01$.

There are few trials examining the impact of TCZ monotherapy on PROs in patients with RA, with the majority of available data limited to the PROs included in the American College of Rheumatology (ACR) core set (PtGA, pain and HAQ-DI). With respect to these PROs, results observed in AMBITION and ADACTA are consistent with those in the ACT-RAY study, in which biologic-naive MTX-IR patients with active RA who switched from MTX to TCZ monotherapy reported improvements $\geq$ MCID in PtGA, pain and HAQ-DI at 24 weeks; improvements were similar between those who switched from MTX to TCZ monotherapy and those who added TCZ to MTX. ${ }^{22}$ However, beyond improvement in the ACR core set components, patients have expressed the importance of alleviating disruptions to work productivity, social functioning, fatigue and the negative mental and emotional effects resulting from this disease. ${ }^{2}$ By evaluating the impact of TCZ on improvement of fatigue and physical, social and mental/emotional well-being measures encompassed in the SF-36, the present study substantially expands the understanding of the efficacy of TCZ improving PROs and patients' HRQOL.

One limitation of this study is the use of ADA monotherapy as the comparator in ADACTA. Although TCZ has similar efficacy whether administered as monotherapy or with MTX, it is well recognised that ADA in combination with MTX is more effective than ADA monotherapy. ${ }^{23} 24$ However, for patients who cannot tolerate MTX, the results presented here suggest that TCZ monotherapy is more effective than ADA monotherapy for improving PROs. Another limitation is the evaluation of PROs only up to 24 weeks; longer studies will be necessary to determine the long-term effects of TCZ monotherapy on PROs. An inherent limitation to trials evaluating PROs is the potential for patient anticipation of improvements due to initiation of new therapy, which may influence reporting of results. Importantly, reported improvements in PROs correlated with significant improvements in CDAI. 
A

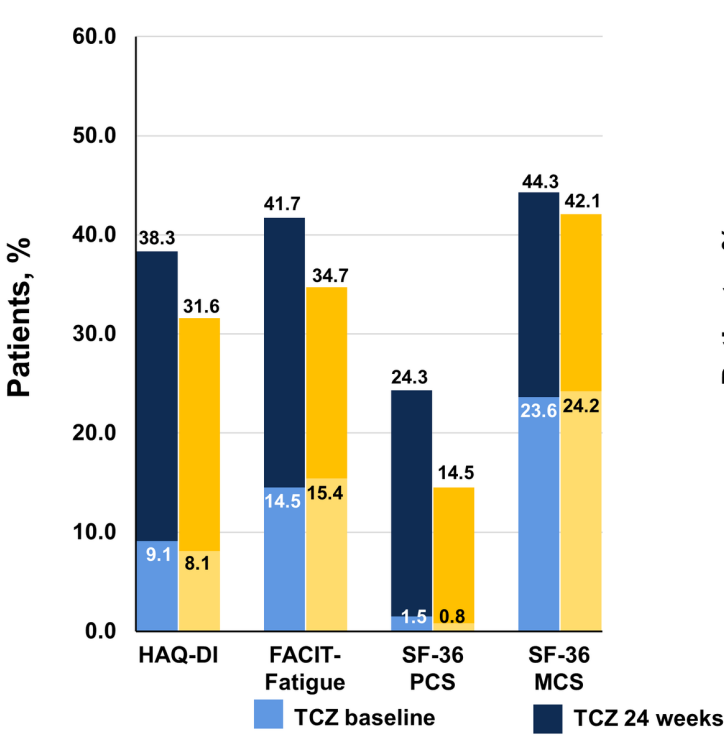

AMBITION

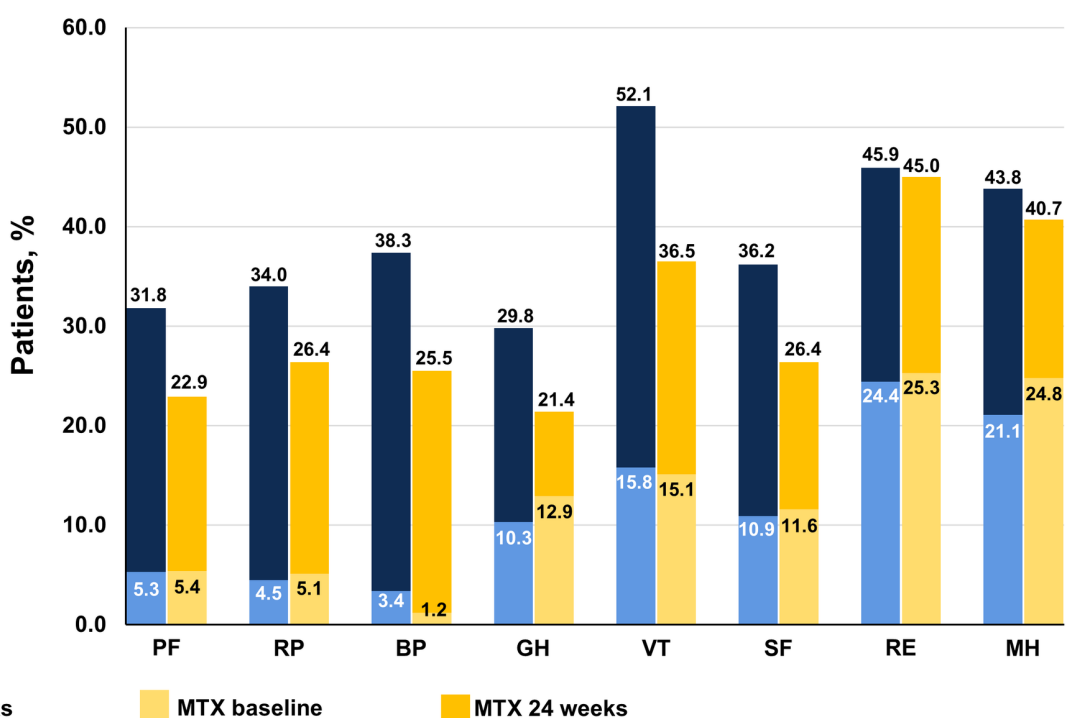

ADACTA

B

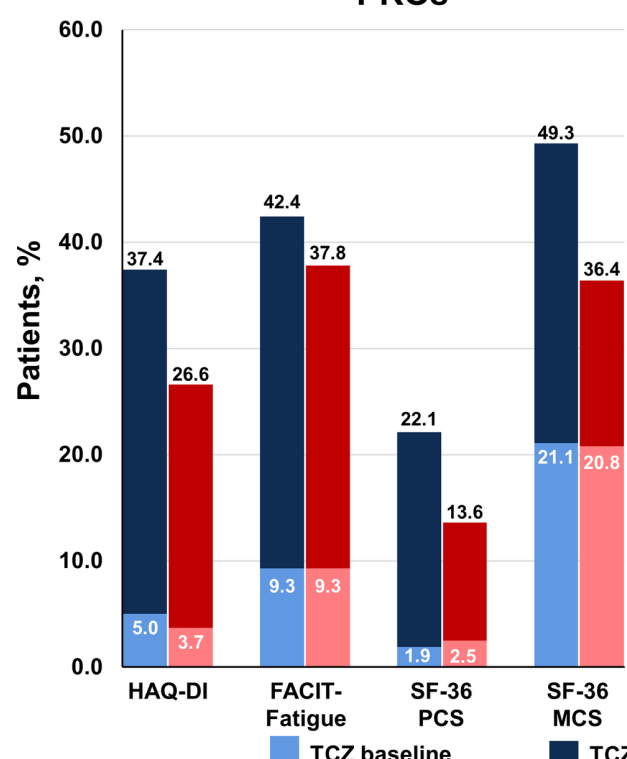

PROs

TCZ 24 weeks

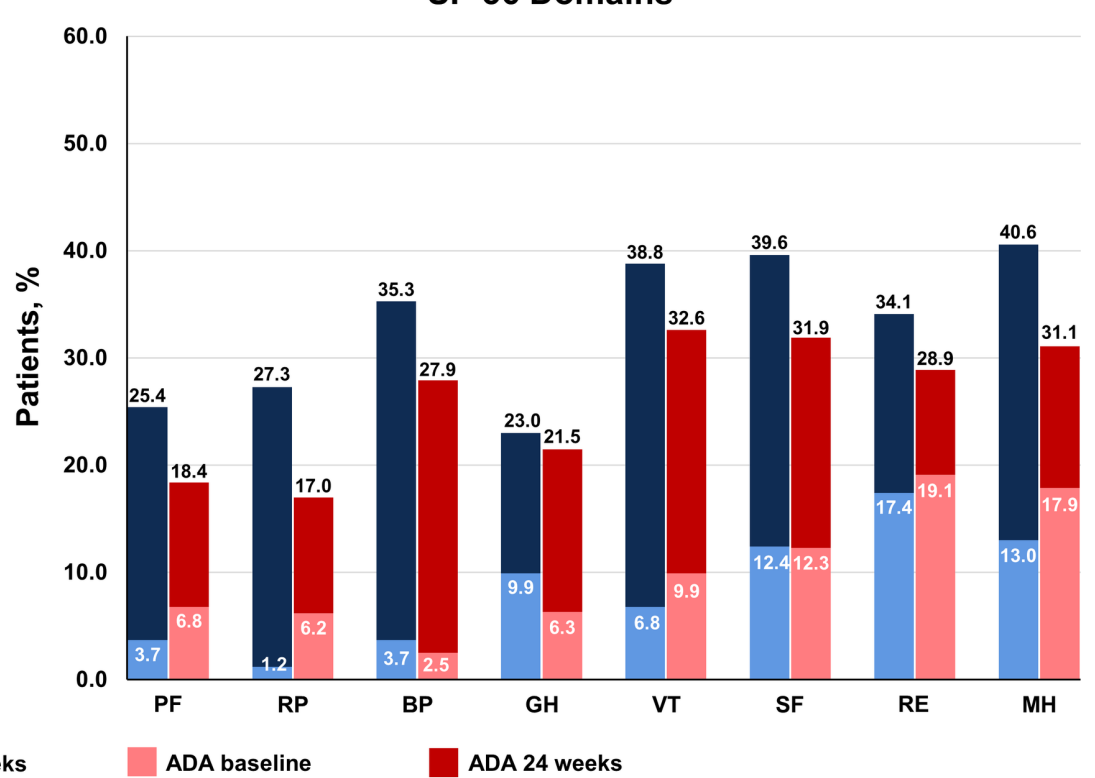

ADA baseline
SF-36 domains

Figure 3 Proportion of patients reporting scores $\geq$ age-matched and gender-matched normative PRO values at baseline and 24 weeks in the (A) AMBITION and (B) ADACTA trial populations. Analyses were performed using the per-protocol population in AMBITION (TCZ, n=265; MTX, n=259) and the intention-to-treat population in ADACTA (TCZ, $n=163 ;$ ADA, $n=162$ ). Normative values were defined as age-matched and gender-matched scores in a non-RA population without comorbid conditions. HAQ-DI: <0.5; FACIT-Fatigue: $\geq 40$; SF-36 PCS/MCS: $\geq 50$; SF-36 domains in the AMBITION population: PF: $\geq 78.8$; RP: $\geq 79.1$; $\mathrm{BP}: \geq 67.4 ; \mathrm{GH}: \geq 68.2$; VT: $\geq 56.6$; SF: $\geq 81.7$; RE: $\geq 85.0$; $\mathrm{MH}: \geq 72.9$; SF-36 domains in the ADACTA population: PF: $\geq 78.3$; $\mathrm{RP}: \geq 79.0$; BP: $\geq 68.1$; GH: $\geq 69.3$; VT: $\geq 58.3$; SF: $\geq 83.4$; RE: $\geq 86.3$; MH: $\geq 75.1$. ADA, adalimumab; BP, bodily pain; FACIT, Functional Assessment of Chronic Illness Therapy; GH, general health; HAQ-DI, Health Assessment Questionnaire Disability Index; MCS, mental component summary; MH, mental health; MTX, methotrexate; PCS, physical component summary; PF, physical functioning; PRO, patient-reported outcome; RA, rheumatoid arthritis; RE, role-emotional; RP, role-physical; SF, social functioning; SF-36, Short Form-36; TCZ, tocilizumab; VT, vitality.

\section{CONCLUSIONS}

Treatment with TCZ, MTX or ADA monotherapy was effective in improving PROs, including HRQOL, in patients with active RA. Although patients receiving MTX or ADA reported improvements across all PROs, TCZ-treated patients reported equivalent or greater improvements.
Overall, TCZ was more effective over 24 weeks than MTX in patients without prior inadequate responses to MTX or TNFis and was more effective as a first-line biologic than ADA in patients for whom continued treatment with MTX was inappropriate. Results of these trials indicate that it is now possible for patients with RA to achieve PRO scores that 
more closely approach those reported by healthy populations.

\section{Author affiliations}

'Division of Immunology and Rheumatology, Stanford University School of Medicine, Palo Alto, California, USA

${ }^{2}$ US Medical Affairs, Immunology, Genentech, Inc., South San Francisco, California, USA

${ }^{3}$ gRED Early Clinical Development, OMNI, Genentech, Inc., South San Francisco, California, USA

${ }^{9}$ PDBB-Biostatistics, Roche, Welwyn Garden City, UK

${ }^{10}$ Division of Rheumatology, Geneva University Hospitals, Geneva, Switzerland

${ }^{11}$ Division of Rheumatology, Allergy and Immunology, University of California San Diego, La Jolla, California, USA

${ }^{12}$ Menzies Institute for Medical Research, University of Tasmania, Hobart, Tasmania, Australia

Acknowledgements The authors acknowledge Kathy Lampl for her critical appraisal of this manuscript. Support for third-party writing assistance, furnished by Elizabeth Ohneck, PhD, of Health Interactions, Inc, was provided by F. Hoffmann-La Roche Ltd/Genentech, Inc. A portion of these data was previously presented at the American College of Rheumatology Annual Meeting; November 11-16, 2016, Washington, DC, USA.

Contributors All authors were involved in the study design and/or collection, analysis and interpretation of the data, provided critical revision of the manuscript and approved the final version to be submitted for publication.

Funding This study was funded by F. Hoffmann-La Roche Ltd/Genentech, Inc. Competing interests VS has received consulting fees from AbbVie, Amgen, AstraZeneca, Biogen, Boehringer Ingelheim, Celltrion, Crescendo Bioscience, F. Hoffmann-La Roche Ltd/Genentech, Inc., GSK, Janssen, Eli Lilly, Merck, Novartis, Pfizer, Regeneron, Samsung, Sanofi and UCB. MM, CB, JP and KT are employees of Genentech, Inc. RF is an employee and shareholder of F. Hoffmann-La Roche Ltd. CG has received research grants and consulting fees from AB2 Bio, AbbVie, Actelion, BMS, Debiopharm, MSD, Novartis, Pfizer, Regeneron, F. Hoffmann-La Roche Ltd, Sanofi and UCB. AK is a consultant for F. Hoffmann-La Roche Ltd/ Genentech, Inc. GJ serves as a speaker, consultant or clinical trialist for Sanofi, AbbVie, Janssen, Pfizer, F. Hoffmann-La Roche Ltd, Eli Lilly, Amgen, BMS and UCB.

Ethics approval The AMBITION and ADACTA study protocols were approved by an ethics committee or institutional review board at each participating center before the start of the study.

Provenance and peer review Not commissioned; externally peer reviewed.

Data sharing statement № additional data are available.

Open Access This is an Open Access article distributed in accordance with the Creative Commons Attribution Non Commercial (CC BY-NC 4.0) license, which permits others to distribute, remix, adapt, build upon this work non-commercially, and license their derivative works on different terms, provided the original work is properly cited and the use is non-commercial. See: http://creativecommons.org/ licenses/by-nc/4.0/

(c) Article author(s) (or their employer(s) unless otherwise stated in the text of the article) 2017. All rights reserved. No commercial use is permitted unless otherwise expressly granted.

\section{REFERENCES}

1. Austad C, Kvien TK, Olsen IC, et al. Sleep disturbance in patients with rheumatoid arthritis is related to fatigue, disease activity, and other patient-reported outcomes. Scand J Rheumatol 2017;46:95-103.

2. Strand V, Khanna D. The impact of rheumatoid arthritis and treatment on patients' lives. Clin Exp Rheumatol 2010;28:32-40.

3. van Vilsteren M, Boot CR, Knol DL, et al. Productivity at work and quality of life in patients with rheumatoid arthritis. $B M C$ Musculoskelet Disord 2015;16:107.

4. Wan SW, He HG, Mak A, et al. Health-related quality of life and its predictors among patients with rheumatoid arthritis. Appl Nurs Res 2016;30:176-83.
5. Deshpande PR, Rajan S, Sudeepthi BL, et al. Patient-reported outcomes: A new era in clinical research. Perspect Clin Res 2011;2:137-44

6. Her M, Kavanaugh A. Patient-reported outcomes in rheumatoid arthritis. Curr Opin Rheumatol 2012;24:327-34.

7. Kalyoncu U, Dougados M, Daurès JP, et al. Reporting of patientreported outcomes in recent trials in rheumatoid arthritis: a systematic literature review. Ann Rheum Dis 2009;68:183-90.

8. Kirwan JR, Hewlett SE, Heiberg T, et al. Incorporating the patient perspective into outcome assessment in rheumatoid arthritis-progress at OMERACT 7. J Rheumatol 2005;32:2250-6.

9. Strand V, Singh JA. Newer biological agents in rheumatoid arthritis: impact on health-related quality of life and productivity. Drugs 2010;70:121-5.

10. Singh JA, Saag KG, Bridges SL, et al. 2015 American College of Rheumatology Guideline for the Treatment of Rheumatoid Arthritis. Arthritis Care Res 2016:68:1-25.

11. Catay E, Bravo M, Rosa J, Soriano ER, et al. Prevalence of biologics monotherapy in a cohort of patients with Rheumatoid Arthritis in daily clinical practice. BMC Musculoskelet Disord 2016;17:110.

12. Pappas DA, Reed GW, Saunders K, et al. Characteristics Associated with Biologic Monotherapy Use in Biologic-Naive Patients with Rheumatoid Arthritis in a US Registry Population. Rheumatol Ther 2015;2:85-96.

13. Dougados M, Kissel K, Sheeran T, et al. Adding tocilizumab or switching to tocilizumab monotherapy in methotrexate inadequate responders: 24-week symptomatic and structural results of a 2-year randomised controlled strategy trial in rheumatoid arthritis (ACTRAY). Ann Rheum Dis 2013;72:43-50.

14. Maini RN, Taylor PC, Szechinski J, et al. Double-blind randomized controlled clinical trial of the interleukin-6 receptor antagonist, tocilizumab, in European patients with rheumatoid arthritis who had an incomplete response to methotrexate. Arthritis Rheum 2006;54:2817-29.

15. Gabay C, Emery P, van Vollenhoven R, et al. Tocilizumab monotherapy versus adalimumab monotherapy for treatment of rheumatoid arthritis (ADACTA): a randomised, double-blind, controlled phase 4 trial. Lancet 2013;381:1541-50

16. Jones G, Sebba A, Gu J, et al. Comparison of tocilizumab monotherapy versus methotrexate monotherapy in patients with moderate to severe rheumatoid arthritis: the AMBITION study. Ann Rheum Dis 2010;69:88-96.

17. Strand V, Burmester GR, Ogale S, et al. Improvements in healthrelated quality of life after treatment with tocilizumab in patients with rheumatoid arthritis refractory to tumour necrosis factor inhibitors: results from the 24-week randomized controlled RADIATE study. Rheumatology 2012;51:1860-9.

18. Strand V, Boers M, Idzerda L, et al. It's good to feel better but it's better to feel good and even better to feel good as soon as possible for as long as possible. Response criteria and the importance of change at OMERACT 10. J Rheumatol 2011;38:1720-7.

19. Webster K, Cella D, Yost K. The Functional Assessment of Chronic Illness Therapy (FACIT) Measurement System: properties, applications, and interpretation. Health Qual Life Outcomes 2003;1:79.

20. Krishnan E, Tugwell P, Fries JF. Percentile benchmarks in patients with rheumatoid arthritis: Health Assessment Questionnaire as a quality indicator (QI). Arthritis Res Ther 2004;6:R505-13.

21. Strand V, Crawford B, Singh J, et al. Use of "spydergrams" to present and interpret SF-36 health-related quality of life data across rheumatic diseases. Ann Rheum Dis 2009;68:1800-4.

22. Dougados M, Kissel K, Sheeran T, et al. Adding tocilizumab or switching to tocilizumab monotherapy in methotrexate inadequate responders: 24-week symptomatic and structural results of a 2-year randomised controlled strategy trial in rheumatoid arthritis (ACTRAY). Ann Rheum Dis 2013;72:43-50.

23. Breedveld FC, Weisman MH, Kavanaugh AF, et al. The PREMIER study: A multicenter, randomized, double-blind clinical trial of combination therapy with adalimumab plus methotrexate versus methotrexate alone or adalimumab alone in patients with early, aggressive rheumatoid arthritis who had not had previous methotrexate treatment. Arthritis Rheum 2006;54:26-37.

24. Strand V, Rentz AM, Cifaldi MA, et al. Health-related quality of life outcomes of adalimumab for patients with early rheumatoid arthritis: results from a randomized multicenter study. J Rheumatol 2012;39:63-72. 